\title{
Periodic attractors for the varactor equation
}

\author{
Michele V. Bartuccelli*, Jonathan H.B. Deane* and Guido Gentile ${ }^{\dagger}$ \\ *Department of Mathematics and Statistics, University of Surrey, Guildford, GU2 7XH, UK. \\ E-mails: m.bartuccelli@surrey.ac.uk, j.deane@surrey.ac.uk \\ ${ }^{\dagger}$ Dipartimento di Matematica, Università di Roma Tre, Roma, I-00146, Italy. \\ E-mail: gentile@mat.unirom3.it
}

\begin{abstract}
We consider a class of differential equations, $\ddot{x}+\gamma \dot{x}+x^{2 p}=f(\boldsymbol{\omega} t)$, with $p \in \mathbb{N}$ and $\boldsymbol{\omega} \in \mathbb{R}^{d}$, describing one-dimensional dissipative systems subject to a periodic forcing. For $p=1$ the equation describes a resistor-inductor-varactor circuit, hence the name 'varactor equation'. We concentrate on the limit cycle described by the trajectory with the same period as the forcing; numerically, for $\gamma$ large enough, it appears to attract all trajectories which remain bounded in phase space. We find estimates for the basin of attraction of this limit cycle, which are good for large values of $\gamma$. Also, we show that the results extend to the case of quasi-periodic forcing, provided the frequency vector $\boldsymbol{\omega}$ satisfies a Diophantine condition - for instance, the Bryuno or the standard Diophantine condition.
\end{abstract}

\section{Introduction}

We consider here the ordinary differential equation

$$
\ddot{x}+\gamma \dot{x}+g(x)=f(\boldsymbol{\omega} t), \quad g(x)=x^{2 p},
$$

where $p \in \mathbb{N}, \boldsymbol{\omega} \in \mathbb{R}^{d}$ is the frequency vector, $f(\boldsymbol{\psi})$ is an analytic quasi-periodic function,

$$
f(\boldsymbol{\psi})=\sum_{\boldsymbol{\nu} \in \mathbb{Z}^{d}} \mathrm{e}^{i \boldsymbol{\nu} \cdot \boldsymbol{\psi}} f_{\boldsymbol{\nu}}
$$

with mean $\langle f\rangle \equiv f_{0}>0$, and where $\gamma>0$ is a real parameter which sets the level of dissipation. This equation with $p=1$ and $d=1$ arises as a description of the behaviour of a simple nonlinear electronic circuit, the resistor-inductor-varactor circuit; travelling wave solutions of the Korteweg-de Vries equation; and the dynamics of ships in beam seas. In the following, for simplicity, we shall refer to (1.1) with $p=1$ as the varactor equation.

For $d>1$ we shall assume a Diophantine condition on $\boldsymbol{\omega}$ like the standard condition

$$
|\boldsymbol{\omega} \cdot \boldsymbol{\nu}| \geq C_{0}|\boldsymbol{\nu}|^{\tau} \quad \forall \boldsymbol{\nu} \in \mathbb{Z}^{d} \backslash\{\mathbf{0}\},
$$

where $|\boldsymbol{\nu}|=\left|\nu_{1}\right|+\ldots+\left|\nu_{d}\right|$, or the weaker Bryuno condition [5]

$$
\sum_{n=0}^{\infty} \frac{1}{2^{n}} \log \frac{1}{\alpha_{n}(\boldsymbol{\omega})}<\infty, \quad \alpha_{n}(\boldsymbol{\omega})=\inf _{|\boldsymbol{\nu}| \leq 2^{n}}|\boldsymbol{\omega} \cdot \boldsymbol{\nu}|
$$

Under this condition on $\boldsymbol{\omega}$ it can be proved that equation (1.1) admits a quasi-periodic attractor characterised by the same frequency vector as the forcing term $[4,1]$. Note that the condition $f_{\mathbf{0}}>0$ is necessary for a quasi-periodic solution with frequency vector $\boldsymbol{\omega}$ to occur: indeed this can happen only if the zeroth-order Fourier component of $g(x)$, which is strictly positive, equals $f_{\mathbf{0}}$. 
Some results concerning equation (1.1) were published in [3]. Here, we extend the analysis in [3], also using the techniques outlined in [1], to prove results in the case of large dissipation $(\gamma \gg 0)$.

As the case of periodic forcing is of more evident practical application, we shall concentrate mainly on that, but the results described below apply to any quasi-periodic forcing, provided that a condition like (1.3) or (1.4) is satisfied by the frequency vector.

The application of the techniques in [1] to equation (1.1) is now less straightforward because solutions can blow up (in finite time, as we will show) and the basin of attraction of periodic solutions (or quasiperiodic solutions, when $d>1$ ) is therefore not the whole of the phase plane - even for large $\gamma$. In the specific case of equation (1.1), we can give a good description of the basin of attraction of the periodic solution by a careful refinement of the construction envisaged in [1]; see section 3 . This provides a complementary result to the analysis performed in [3], where the set of initial data generating solutions that blow up was extensively studied. In the case in which the function $f$ is of definite sign (hence positive, as $\langle f\rangle>0$ ), we also construct a positively invariant set containing the attracting periodic orbit which allows us, together with the results of section 3, to obtain a larger set estimating the basin of attraction (with area growing linearly in $\gamma$ ); cf. Theorem 1 in section 4.

Finally we show that there are unbounded solutions which blow up in finite time, and we discuss the implications for the varactor equation in [3]; cf. Theorem 2 in section 5. In particular, this allows us to settle a problem left open in [3], about the blow-up time of the unbounded solutions.

The rest of the paper is organised as follows. In section 2 we briefly review the results on equation (1.1) in $[1,5,3]$, which will be used in what follows. In section 3 we give the construction for $g(x)=x^{2 p}$, with particular emphasis on $p=1$, and we look for an estimate of the basin of attraction of the (quasi-)periodic solution. This construction improves that given in [3] for general nonlinearities. Then, in section 4, we use the techniques of [3] and [1] to improve further the estimate of the basin of attraction. In section 5 we show that in the case $g(x)=x^{2 p}$, there are unbounded solutions which blow up in finite time. Finally in section 6 we mention some open problems.

\section{Existence of quasi-periodic attractors}

In this section we review the results found in $[1,5,3]$. We start with a result on the existence of quasi-periodic attractors, proved - for more general nonlinearities $g(x)$ - in [1], section 5, in the case of Diophantine frequency vectors, that is frequency vectors satisfying the standard Diophantine condition (1.3), and in [5], section 4, in the case of Bryuno frequency vectors, that is frequency vectors satisfying the Bryuno condition (1.4). As the second condition implies the first one, we state the result directly for Bryuno frequency vectors.

Theorem 1 Consider equation (1.1), with $f$ analytic given by (1.2), and with $\boldsymbol{\omega}$ satisfying the Bryuno condition (1.4). Assume that $f_{\mathbf{0}}$ is strictly positive. Then there exists $\gamma_{0}$ such that for $\gamma>\gamma_{0}$ there is a unique quasi-periodic solution $x_{0}(t)$ which has the same frequency vector as $f$ and reduces to $c_{0}=\left(f_{0}\right)^{1 / 2 p}$ in the limit $\gamma \rightarrow \infty$. Furthermore, there exists $\gamma_{1} \geq \gamma_{0}$ such that for $\gamma>\gamma_{1}$ there is a neighbourhood $\mathcal{I}$ of the point $\left(c_{0}, 0\right)$ with the property that all trajectories starting in $\mathcal{I}$ are attracted to the orbit described by $x_{0}(t)$ in the plane.

It should be noted that the estimates provided in [1] hold for very general $g(x)$, and so they turn out to be very poor if applied naively to specific cases - for instance, the varactor equation.

The second result that we need is Theorem 2.15 of [3], section 2, for the varactor equation. Define the set $\mathcal{B}$ and $\mathcal{B}_{7}$ as in the statements of Theorem 2.4 and, respectively, of Lemma 2.5 of [3] - cf. figure 2 in [3]. Then the following result holds.

Theorem 2 All solutions initially in $\mathcal{B}$ remain in $\mathcal{B}$, eventually entering $\mathcal{B}_{7}$, where they remain and grow without limit. 
The above result allows us to confine ourselves to a substantially limited region of phase plane, $\mathcal{B}^{c}$, the complement of $\mathcal{B}$, in seeking attractors and to describe their basins of attraction. For $\gamma$ large enough the existence of a quasi-periodic attractor is assured by Theorem 1. In agreement with Theorem 2, its projection onto the $(x, \dot{x})$ plane is located inside the set $\mathcal{B}^{c}$. As pointed out, the estimates provided in [1] work in general, but, exactly because of this fact, are not optimal, and turn out to be very deceptive if applied to concrete examples. In next section, we shall see how to obtain better estimates in the specific case of the equation under study.

\section{Application to the varactor equation, and generalisations}

The construction of the neighbourhood $\mathcal{I}$ of Theorem 1 can be improved in concrete examples. Here we consider explicitly the case of even monomials $g(x)=x^{2 p}$, with particular emphasis on $p=1$ (the varactor equation). Hence we discuss explicitly the case $p=1$, but the discussion can easily be extended to all $p \geq 1$.

Let us fix $p=1$, and write $x(t)=x_{0}(t)+\xi(t)$, where $x_{0}(t)$ is the quasi-periodic solution which has the same frequency vector $\boldsymbol{\omega}$ as the forcing.

We define the functions $F(\xi, x)$ and $R(\xi, t)$ as in [1], section 3 , so that

$$
\xi F(\xi, x)=\xi^{2}+2 x \xi, \quad R(\xi, t)=\frac{\xi+2 x_{0}(t)}{\xi+2 c_{0}}=1+2 \frac{x_{0}(t)-c_{0}}{\xi+2 c_{0}}
$$

where $c_{0}=\sqrt{f_{\mathbf{0}}}$, with $f_{\mathbf{0}}>0$. For $\gamma$ large enough, that is for $\varepsilon=1 / \gamma$ small enough, one has

$$
\left|x_{0}(t)-c_{0}\right|<C_{1} \varepsilon
$$

for a suitable constant $C_{1}$. This follows from the analysis performed in $[4,1]$; see in particular the first lines of the proof of Theorem 4 in [1].

It is easy to see that one has $\lim _{\xi \rightarrow 0} R(\xi, t)=1+O(\varepsilon)$, so that for $\varepsilon$ small enough one has $R_{1}<$ $R(\xi, t)<R_{2}$, for two suitable positive constants $R_{1}$ and $R_{2}$.

Then we can rescale time and the dependent variables by setting

$$
\tau=\int_{0}^{t} \mathrm{~d} t \sqrt{R\left(\xi\left(t^{\prime}\right), t^{\prime}\right)}, \quad \xi(t)=v(\tau(t)), \quad y(t)=\sqrt{R(\xi(t), t)} w(\tau(t)) .
$$

This transforms the system (1.1) into

$$
\left\{\begin{array}{l}
v^{\prime}=w \\
\left.w^{\prime}=-\frac{w}{\sqrt{R}}\left(\gamma+\frac{R^{\prime}}{2 \sqrt{R}}\right)-v F\left(v, c_{0}\right)\right),
\end{array}\right.
$$

with the primes denoting differentiation with respect to $\tau$, and

$$
v F\left(v, c_{0}\right)=v^{2}+2 c_{0} v
$$

Hence, if we set $\bar{\gamma}=\left(\gamma+R^{\prime} / 2 \sqrt{R}\right) / \sqrt{R}$, we can interpret the system (3.4) as a Hamiltonian system with hamiltonian

$$
H(v, w)=\frac{1}{2} w^{2}+U(v), \quad U(v)=\frac{1}{3} v^{3}+c_{0} v^{2},
$$

in the presence of a friction term with non-constant dissipation coefficient $\bar{\gamma}$. If we neglect the friction term, that is if we put $\bar{\gamma}=0$, then the system becomes the Hamiltonian system

$$
\left\{\begin{array}{l}
v^{\prime}=w \\
w^{\prime}=-v^{2}-2 c_{0} v
\end{array}\right.
$$


which admits a stable equilibrium point $P_{1}=(0,0)$, an unstable equilibrium point $P_{2}=\left(-2 c_{0}, 0\right)$ and the separatrix $\Gamma$ with equation

$$
w= \pm \sqrt{2\left(H\left(-2 c_{0}, 0\right)-U(v)\right)}, \quad H\left(-2 c_{0}, 0\right)=\frac{4 c_{0}^{3}}{3},
$$

which contains a homiclinic orbit to the right of $P_{2}$. Consider the open set $\mathcal{S}_{1}$ containing the point $P_{1}$ and with boundary given by (the closure of) the homoclinic orbit in $\Gamma$. Any level curve with energy $V(0)<E<V\left(-2 c_{0}\right)$ contains a bounded connected component, internal to $\mathcal{S}_{1}$, which is a closed orbit $\mathcal{C}_{E}$ for the system (3.7). Denote by $\mathcal{C}$ the closed curve $\mathcal{C}_{E}$ which intersects the negative $v$-axis at $v=-2 c_{0}+C_{2} \varepsilon^{\beta}$, for suitable constants $C_{2}$ and $\beta$ to be fixed, and $\mathcal{S}$ the open set containing $P_{1}$ with boundary $\mathcal{C}$.

From (3.1), one has

$$
|R(\xi, t)-1|<\frac{C_{1}}{C_{2}} \varepsilon^{1-\beta},
$$

so that $R(\xi, t) \rightarrow 1$ as $\varepsilon \rightarrow 0$, provided $\beta<1$; for instance one can take $\beta=1 / 2$. Then, if $\gamma$ is large enough, for any point $(v, w) \in \overline{\mathcal{S}}$ one has

$$
\left|R^{\prime}(\xi, t)\right| \leq\left|\frac{2 \xi^{\prime}\left(c_{0}-x_{0}\right)}{\left(\xi+2 c_{0}\right)^{2}}\right|+\left|\frac{2 x_{0}^{\prime}}{\xi+2 c_{0}}\right| \leq C_{3} \varepsilon^{1-2 \beta}+C_{4} \varepsilon^{1-\beta},
$$

for suitable constants $C_{3}$ and $C_{4}$, so that, by also using that $\xi=v$ and

$$
\left|\xi+2 c_{0}\right| \geq C_{2} \varepsilon^{\beta}
$$

for all $(v, w) \in \overline{\mathcal{S}}$, one obtains

$$
\left|\gamma-\gamma^{\prime}\right| \leq\left|\frac{R^{\prime}}{2 R}\right| \leq C_{5} \varepsilon^{1-2 \beta} \leq C_{5} \varepsilon^{2(1-\beta)} \gamma<\gamma
$$

for a suitable constant $C_{5}$, hence $\gamma^{\prime}>0$ for $\gamma$ large enough. Therefore we can conclude that $\overline{\mathcal{S}}$ is positively invariant, and we can apply Barbashin-Krasovsky's theorem [6] to conclude that the equilibrium point $P_{1}$ is asymptotically stable and the set $\mathcal{S}$ is contained inside its basin of attraction.

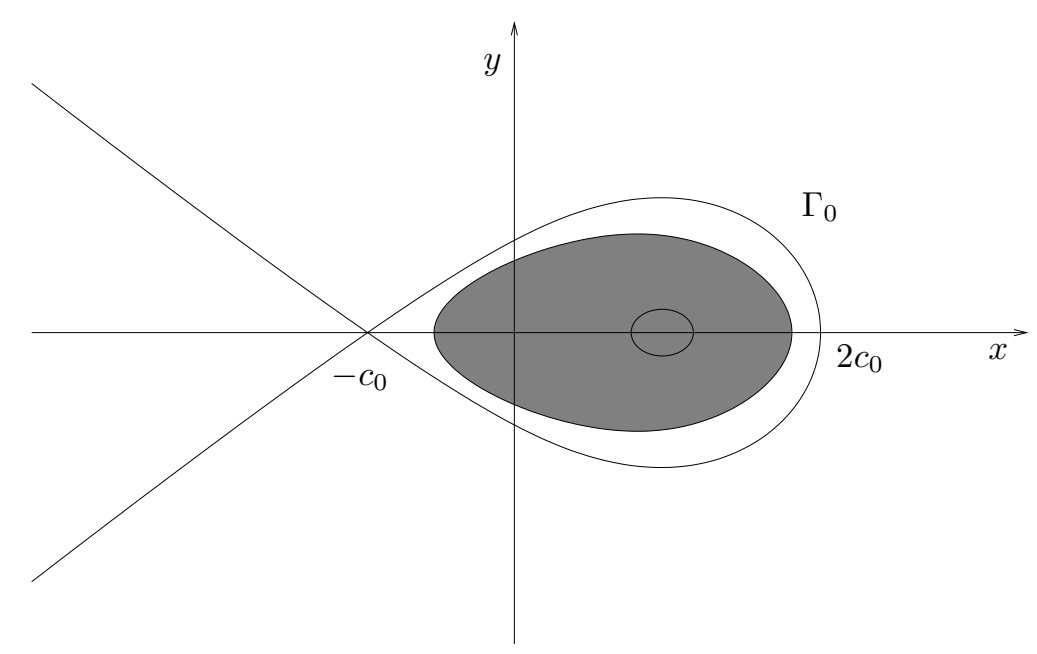

Figure 1: Estimate of the basin of attraction (grey set $\mathcal{D}$ ) of the quasi-periodic attractor for large $\gamma$. The small closed curve inside the grey set represents the attractor in the case of periodic forcing $(d=1)$, when the attractor is a limit cycle. The curve $\Gamma_{0}$ is the separatrix of the system described by the differential equation $\ddot{x}+x^{2}=c_{0}^{2}$. 
If we go back to the original coordinates, we see that, for $\gamma$ large enough, there is a quasi-periodic solution $x_{0}(t)$ which moves very close to the point $\left(c_{0}, 0\right)$ (within a distance of order $\varepsilon=1 / \gamma$ ); see figure 1. Its basin of attraction contains a large set $\mathcal{D}$ whose boundary is at a distance of order $\varepsilon^{\beta}$ from the separatrix $\Gamma_{0}$ of the system described by the equation $\ddot{x}+x^{2}=c_{0}^{2}$, that is from the curve $y= \pm \sqrt{2\left(2 c_{0}^{3}-x^{3}+3 c_{0}^{2} x\right) / 3}$.

\section{Improved estimates for the basins of attraction}

We start by constructing an invariant set $\mathcal{A}$, valid for all $p \in \mathbb{N}$, which has the property that its area is $O(\gamma)$. We need the additional assumption that $f(\boldsymbol{\omega} t)>0$ for all $t$. The construction is surely not optimal but is included because the set $\mathcal{A}$ is, amongst several sets constructed, one which grows with $\gamma$ in the vertical direction. Constructing invariant sets containing the attracting orbit for $\gamma$ large is not difficult, but it was less obvious how to find one that grows with $\gamma$. The construction follows very closely the ideas exploited in [3].

Rewrite (1.1), with $g(x)=x^{2 p}$, as

$$
\left\{\begin{array}{l}
\dot{x}=y \\
\dot{y}=f(\boldsymbol{\omega} t)-\gamma y-x^{2 p}
\end{array}\right.
$$

so that the vector field generated by the differential equation is defined by $\phi(t)=\left(y, f(\boldsymbol{\omega} t)-\gamma y-x^{2 p}\right)$. Let $f^{2 p} \leq f(\boldsymbol{\omega} t) \leq F^{2 p}$. Note that in this and the following section, $f$ is just a constant and not the function $t \rightarrow f(\boldsymbol{\omega} t)$. We adopt this notation here to conform to that used in the analysis in [3]. For the same reason we denote in boldface vectors in $\mathbb{R}^{2}$, so that $\boldsymbol{a} \cdot \boldsymbol{b}$ denotes the scalar product in $\mathbb{R}^{2}$.

The two vector fields, $\phi_{F}=\left(y, F^{2 p}-\gamma y-x^{2 p}\right)$ and $\phi_{f}=\left(y, f^{2 p}-\gamma y-x^{2 p}\right)$, have no explicit time-dependence and also have the property that for all $t, \phi(t)=\mu \phi_{F}+(1-\mu) \phi_{f}$ where $\mu \in[0,1]$ is a (time-dependent) scalar.

Following [3], we let the boundary of invariant set $\mathcal{A}$ be a hexagon GHIJKL whose edges are straight lines, except for HI and KL. GH and JK are horizontal and LG and IJ are vertical - see figure 2. The co-ordinates of points $\mathrm{H}$ and $\mathrm{K}$ are $\left(0, y_{H}\right)$ and $\left(0, y_{K}\right)$ respectively. The dotted curves in figure 2 are $P_{F}: y=\left(F^{2 p}-x^{2 p}\right) / \gamma$ (upper) and $P_{f}: y=\left(f^{2 p}-x^{2 p}\right) / \gamma$ (lower). As shown in [3], only in the region between these curves is the sign of $\dot{y}$ ambiguous; above $P_{F}, \dot{y}<0$ and below $P_{f}, \dot{y}>0$. Provided that $y_{H} \geq F^{2 p} / \gamma$, which will turn out to be automatically satisfied, $\phi(t)$ will be into $\mathcal{A}$ along GH, and point $\mathrm{J}$ being below $P_{f}$ guarantees that $\phi(t)$ will always be into KJ, both results holding for all $t$. Furthermore, the sign of $\dot{x}=y$ guarantees that $\phi(t)$ will be into LG and IJ, again for all $t$. It therefore remains to define the sides $\mathrm{HI}$ and $\mathrm{KL}$ in such a way that it is possible to prove that $\phi(t)$ is into them for all $t$.

Let us define HI by $y=\lambda_{1}\left(F^{2 p}-x^{2 p}\right)$ with $\lambda_{1} \in \mathbb{R}$ positive; then the normal pointing into $\mathcal{A}$, $\boldsymbol{n}_{1}=\left(-2 p \lambda_{1} x^{2 p-1},-1\right)$. In order to prove that $\boldsymbol{\phi}(t)$ is into HI for all time, we need only to show that $\boldsymbol{n}_{1} \cdot \boldsymbol{\phi}_{F} \geq 0$ for $x \in[0, F]$. We have $\boldsymbol{n}_{1} \cdot \boldsymbol{\phi}_{F}=\left[F^{2 p}-x^{2 p}\right]\left[\lambda_{1}\left(\gamma-2 p \lambda_{1} x^{2 p-1}\right)-1\right]$ and, since $F^{2 p}-x^{2 p} \geq 0$, and the second bracket in the scalar product reaches its minimum value over $[0, F]$ at $x=F$, we require

$$
2 p F^{2 p-1} \lambda_{1}^{2}-\gamma \lambda_{1}+1 \leq 0,
$$

in order for the scalar product to be non-negative. Solving this quadratic in $\lambda_{1}$ and choosing the larger solution gives

$$
\lambda_{1}=\frac{\gamma}{4 p F^{2 p-1}}\left[1+\sqrt{1-\frac{8 p F^{2 p-1}}{\gamma^{2}}}\right]
$$

provided that $\lambda_{1} \in \mathbb{R}$. This is true for $\gamma^{2} \geq 8 p F^{2 p-1}$. We now show that this condition on $\gamma$ also forces $y_{H}=\lambda_{1} F^{2 p} \geq F^{2 p} / \gamma$, or $\gamma \lambda_{1} \geq 1$, to be satisfied. Letting $q=4 p F^{2 p-1}$, the condition for $\lambda_{1}$ to be real is $\gamma^{2} / q \geq 2$; using this in the definition of $\lambda_{1}$ gives $\gamma \lambda_{1}=\left(\gamma^{2} / q\right)\left(1+\sqrt{1-2 q / \gamma^{2}}\right) \geq$ 


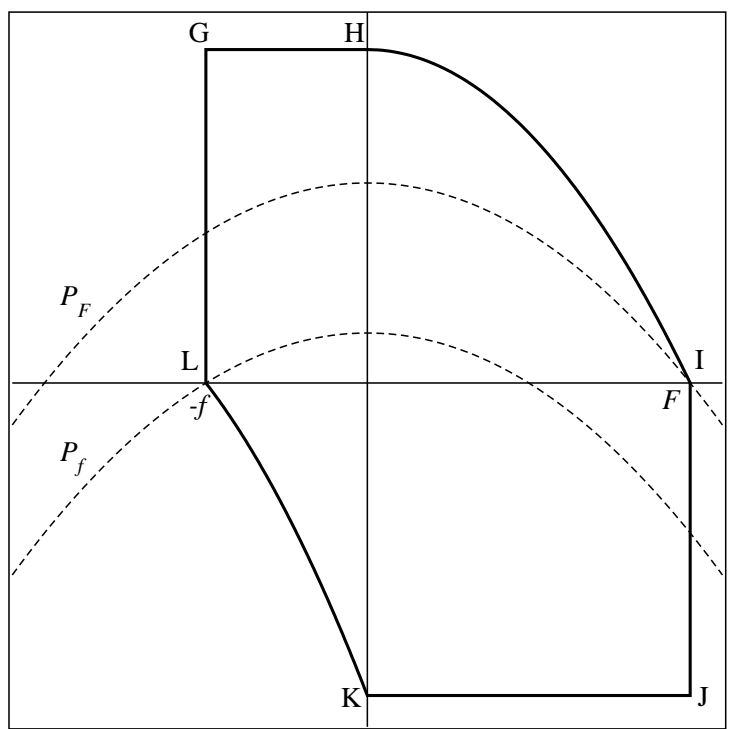

Figure 2: The invariant set $\mathcal{A}$, whose vertices are G, H, I, J, K and L. Also shown as dotted lines are the curves $P_{F}: y=\left(F^{2 p}-x^{2 p}\right) / \gamma$ and $P_{f}: y=\left(f^{2 p}-x^{2 p}\right) / \gamma$.

$2\left(1+\sqrt{1-2 q / \gamma^{2}}\right) \geq 1$, which is clearly true. Hence, boundary HI has been constructed in such a way that $\phi(t)$ is into it for all time. Note that $\lambda_{1} \sim \gamma /\left(2 p F^{2 p-1}\right)$ as $\gamma \rightarrow \infty$, and so $y_{H}=\lambda_{1} F^{2 p}=O(\gamma)$.

We now define KL by $y=\lambda_{2}\left(f^{2 p}-(x+2 f)^{2 p}\right)$ where $\lambda_{2} \in \mathbb{R}>0$. This has inward normal $\boldsymbol{n}_{2}=\left(2 p \lambda_{2}(x+2 f)^{2 p-1}, 1\right)$ and the inequality we need to consider now is

$$
\boldsymbol{\phi}_{f} \cdot \boldsymbol{n}_{2}=\lambda_{2}\left[(x+2 f)^{2 p}-f^{2 p}\right]\left[\gamma-2 p \lambda_{2}(x+2 f)^{2 p-1}\right]+f^{2 p}-x^{2 p} \geq 0
$$

for $x \in\left[-f, x_{K}\right]$ where $x_{K}$, satisfying $F \geq x_{K}>-f$, is to be defined. The first term consists of a product of two terms, the first of which is positive for $x \in(-f, \infty)$ and the second of which is a monotonically decreasing function of $x$ for $x \in \mathbb{R} \backslash\{-2 f\}$; it is zero at $x=x_{0}=\left(\gamma / 2 p \lambda_{2}\right)^{1 /(2 p-1)}-2 f$. Choosing $x_{K}=x_{0}=0$ ensures that the product term in (4.4) is non-negative and gives

$$
\lambda_{2}=\frac{\gamma}{2 p(2 f)^{2 p-1}} .
$$

The last term in inequality (4.4) is non-negative for $x \in[-f, f]$ and so the entire scalar product is nonnegative for $\lambda_{2}$ as given above, provided that $x_{K}=0$. We also require point $\mathrm{J}$ to be below $p_{f}$. The $y$-co-ordinate of $\mathrm{J}=y_{K}=\lambda_{2}\left(f^{2 p}-(2 f)^{2 p}\right)$ and so this condition becomes

$$
\gamma^{2} \geq \frac{p\left(F^{2 p}-f^{2 p}\right)}{f\left(1-4^{-p}\right)} .
$$

We can now state the following result.

Theorem 3 Define $\mathcal{A}$ as the hexagonal set whose vertices are $G, H, I, J, K$ and $L$, where

$$
\begin{gathered}
G=\left(-f, \lambda_{1} F^{2 p}\right), \quad H=\left(0, \lambda_{1} F^{2 p}\right), \quad I=(F, 0), \\
J=\left(F,-\lambda_{2} f^{2 p}\left(4^{p}-1\right)\right), \quad K=\left(0,-\lambda_{2} f^{2 p}\left(4^{p}-1\right)\right), \quad L=(-f, 0),
\end{gathered}
$$

and where $\lambda_{1}$ and $\lambda_{2}$ are given by equations (4.3) and (4.4), respectively. Let the edges LG, GH, IJ and $J K$ of $\mathcal{A}$ be straight lines and let HI be given by $y=\lambda_{1}\left(F^{2 p}-x^{2 p}\right)$ and $K L$, by $y=\lambda_{2}\left(f^{2 p}-(x+2 f)^{2 p}\right)$. 
Then, provided that

$$
\gamma^{2} \geq \max \left(8 p F^{2 p-1}, \frac{p\left(F^{2 p}-f^{2 p}\right)}{f\left(1-4^{-p}\right)}\right),
$$

set $\mathcal{A}$ is an invariant set containing the limit cycle described by the solution $x_{0}(t)$ in the plane.

Figure 3 compares the set GHIJKL with $\mathcal{B}^{c}$ for $p=1,2$ and 3 .
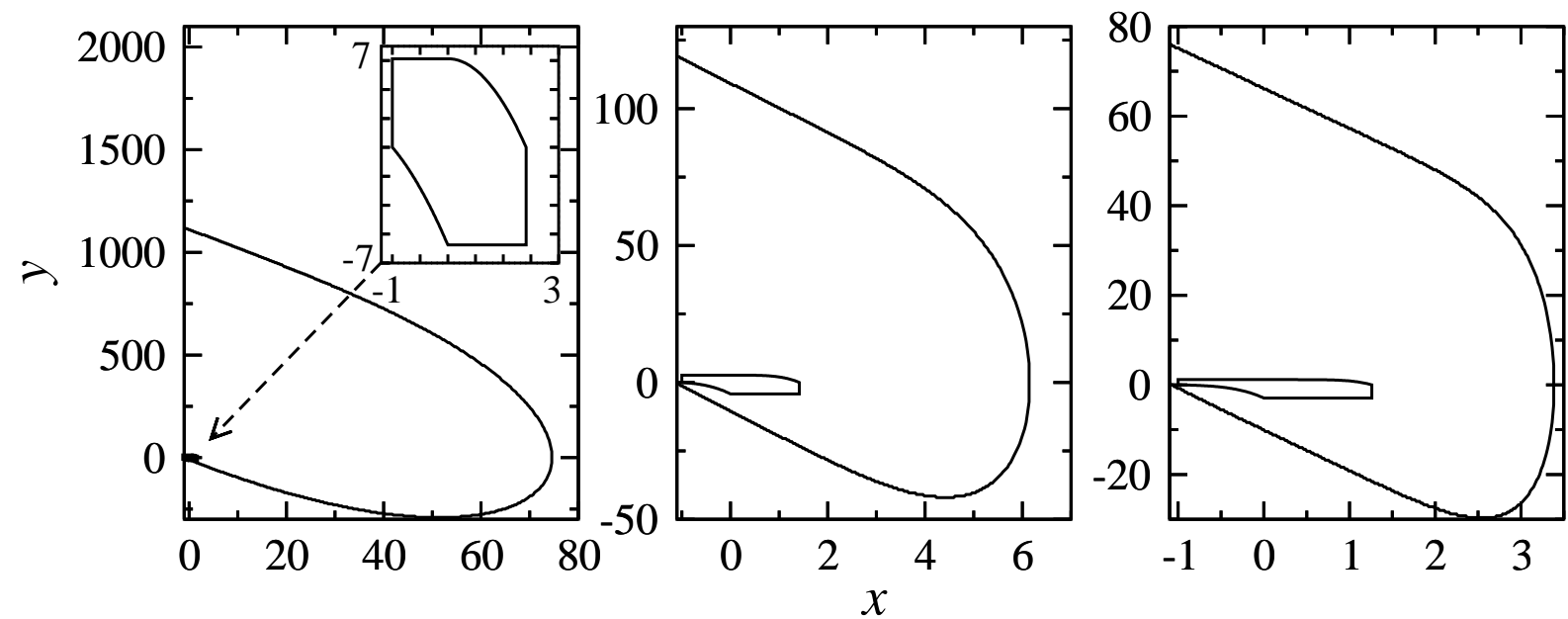

Figure 3: The set $\mathcal{A}$ compared to the set $\mathcal{B}^{c}$ (cf. [3]) with $1 \leq f(t)=(5+3 \sin t) / 2 \leq 4, \gamma=9$ and $p=1$ (left), $p=2$ (middle) and $p=3$ (right). The set $\mathcal{B}$ is defined such that all trajectories starting in $\mathcal{B}$ remain there, hence $\mathcal{B}^{c}$ provides an estimate for the basin of attraction of the periodic solution, $x_{0}(t)$.

The existence of the set $\mathcal{A}$ having the properties stated in Theorem 3 allows us to improve the estimate $\mathcal{D}$ of the basin of attraction found in section 3 for the varactor equation studied in [3], that is for the system (1.1), where $p=1$ and $f(\boldsymbol{\omega} t)=\alpha+\beta \sin t$, with $\alpha>0$ and $|\beta|<\alpha$. Define $\mathcal{D}_{0}=\mathcal{D} \cup \mathcal{A}$. First note that both $\mathcal{D}$ and $\mathcal{A}$ are proper subsets of $\mathcal{D}_{0}$, as follows easily from the remark that $\mathcal{D}_{0}$ is inside the separatrix $\Gamma_{0}$ (cf. the end of section 3), and at a distance $O(1 / \sqrt{\gamma})$ from it. The inclusion $\mathcal{D} \subsetneq \mathcal{D}_{0}$ is obvious as $\mathcal{A}$ grows as $\gamma$ in the vertical direction. The inclusion $\mathcal{A} \subsetneq \mathcal{D}_{0}$ follows from the fact that the separatrix intersects the $x$-axis in $x=-c_{0}$ and $x=2 c_{0}$, with $c_{0}=\sqrt{\alpha}$; hence $x_{L}=-\sqrt{\alpha-\beta}>-c_{0}$ and $x_{I}=\sqrt{\alpha+\beta} \leq \sqrt{2 \alpha}<2 c_{0}$. Then the set $\mathcal{D}_{1}:=\mathcal{A} \backslash(\mathcal{A} \cap \mathcal{D})$ is non-empty - see figure 4. Furthermore a trajectory starting in $\mathcal{D}_{1}$ can leave $\mathcal{D}_{1}$ only by entering $\mathcal{D}_{0}$ (by the invariance of $\mathcal{A}$ ). This means that all trajectories starting in $\mathcal{D}_{0}$ are attracted by the limit cycle described by $x_{0}(t)$.

We emphasise, both here and in [3], the fact that $d=1$ (so that $f(\boldsymbol{\omega} t)$ is a periodic function) plays no role. Indeed quasi-periodic forcing could be considered as well, and the same results would apply.

\section{Blow up in finite time}

We prove finite-time blow up for the system (1.1) by first finding an invariant set $\mathcal{J}$ (Lemma 1 ) and then constructing an invariant subset, $\mathcal{S}\left(-X_{0}\right)$, of $\mathcal{J}$, the latter construction being carried out in Lemma 2 . Within $\mathcal{S}\left(-X_{0}\right)$, a differential inequality must hold and we show in Theorem 4 that all solutions of this differential inequality must blow up in finite time.

Lemma 1 Define $h(x)=2 p x^{2 p-1}\left(F^{2 p}-x^{2 p}\right)-\gamma^{2}\left(F^{2 p}-f^{2 p}\right)$ and the set

$$
\mathcal{J}=\left\{(x, y) \mid x \leq-\xi,\left(F^{2 p}-x^{2 p}\right) / \gamma \leq y \leq 0\right\}
$$

where $-\xi$ is the root of $h(x)$ with $-\xi<-F$. Then $\xi$ as defined exists uniquely and $\mathcal{J}$ is an invariant set. 


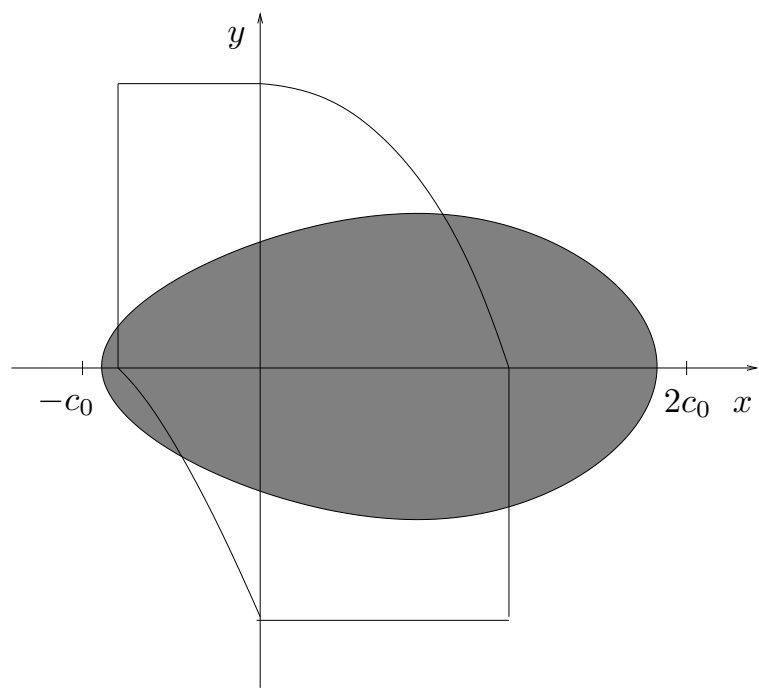

Figure 4: Comparison between the set $\mathcal{D}$ (shaded grey) constructed in section 3 and the set $\mathcal{A}$ defined in Theorem 3. For $f(\boldsymbol{\omega} t)=\alpha+\beta \sin t$ one has $c_{0}=\sqrt{\alpha}, f=\sqrt{\alpha-\beta}$ and $F=\sqrt{\alpha+\beta}$. The boundary of $\mathcal{D}$ intersects the the $x$-axis at $x=x_{1}=-c_{0}+r_{1}$ and $x=x_{2}=2 c_{0}-r_{2}$, where both $r_{1}$ and $r_{2}$ are positive and of order $O(1 / \gamma)$. Hence for $\gamma$ large enough one has $x_{1}<f$ and $x_{2}>F$.

Proof. The set $\mathcal{J}$ is clearly absorbing along its horizontal, $y=0, x \leq-\xi$, and vertical, $x=-\xi$, $\left(F^{2 p}-\xi^{2 p}\right) / \gamma \leq y \leq 0$ boundaries. We therefore only need to prove that it is also absorbing along the curved boundary, $y=\left(F^{2 p}-x^{2 p}\right) / \gamma$ for $x \leq-\xi$. The appropriate normal here is $\boldsymbol{n}=\left(2 p x^{2 p-1} / \gamma, 1\right)$ and so we require $\boldsymbol{n} \cdot \boldsymbol{\phi}_{f} \geq 0$, which is equivalent to proving that $h(x) \geq 0$, for $x \leq-\xi$.

To this end, first note some elementary properties of $h(x)$ : (i) $h(-F)=-\gamma^{2}\left(F^{2 p}-f^{2 p}\right)<0$; (ii) $h(x)$ has exactly three stationary points for $x \in \mathbb{R}: h^{\prime}(x)=0$ for $x=0$ and $x=x_{ \pm}= \pm F[(2 p-1) /(4 p-1)]^{1 / 2 p}$; (iii) $h^{\prime \prime}\left(x_{-}\right)>0$; and (iv) $h(x) \rightarrow \infty$ as $x \rightarrow-\infty$. From these, it becomes clear that $-F<x_{-}<0$ and $h\left(x_{-}\right)$is a minimum; and, in the light of (i) and (iv), $h(x)$ has exactly one real root, $-\xi, \in(-\infty,-F)$. Hence, $h(x) \geq 0$ for $x \leq-\xi$ and the invariance of set $\mathcal{J}$ is proved.

We now define, for $x \leq-X_{0}$, a curve $G(x, y): y=-b\left(-X_{0}-x\right)^{\rho}$ with $-X_{0} \leq-\xi<0$, and $\rho$ and $b>0$ to be specified. In order that $\mathcal{S}\left(-X_{0}\right) \subset \mathcal{J},-X_{0} \leq-\xi$ and $b>0$. In the proof of Theorem 4 below, it will be required that $\mathcal{S}\left(-X_{0}\right)$ has infinite area for all $p \in \mathbb{N}$, and hence $\rho<2-$ otherwise, the curves $\left(F^{2 p}-\xi^{2 p}\right) / \gamma$ and $G$ could intersect at some finite $x<-\xi$ when $p=1$. In order that solutions blow up in finite time, $\rho>1$ will also be required: hence, we choose $\rho=3 / 2$.

The proof of the invariance of $\mathcal{S}$ now follows.

Lemma 2 The set

$$
\mathcal{S}\left(-X_{0}\right)=\left\{(x, y) \mid x \leq-\xi, U \geq y \geq\left(F^{2 p}-x^{2 p}\right) / \gamma\right\}
$$

where

$$
U=\left\{\begin{array}{cl}
-b\left(-X_{0}-x\right)^{3 / 2} & x \leq-X_{0} \\
0 & \text { otherwise }
\end{array}\right.
$$

with $-X_{0} \leq-\xi$ and $b$ sufficiently small, is an invariant subset of $\mathcal{J}$, and contains points $(x, y)$ with $y \rightarrow-\infty$.

Proof. The fact that the vector field is into all the boundaries of $\mathcal{S}$ except $y=-b\left(-X_{0}-x\right)^{3 / 2}$ has been proved in Lemma 1 ; therefore, we only need to consider the boundary $y=-b\left(-X_{0}-x\right)^{3 / 2}$. The 
appropriate normal is that which points into $\mathcal{S}$, this being $\left.\boldsymbol{n}=(3 b / 2) \sqrt{-X_{0}-x},-1\right)$. The correct choice for the vector field here is $\phi_{F}=\left(y, F^{2 p}-\gamma y-x^{2 p}\right)$, so that

$$
\boldsymbol{n} \cdot \boldsymbol{\phi}_{F}=-b\left(-X_{0}-x\right)^{3 / 2}\left[(3 b / 2) \sqrt{-X_{0}-x}+\gamma\right]+x^{2 p}-F^{2 p} \geq 0, \quad x \leq-X_{0} .
$$

Since $-X_{0}-x \geq 0$, we can substitute $v^{2}=-X_{0}-x$, giving

$$
L(v)=\left(X_{0}+v^{2}\right)^{2 p}-F^{2 p}-b v^{3}(3 b v / 2+\gamma) \geq 0, \quad v \geq 0 .
$$

Let $l(x)=x^{p}$; then the mean value theorem states that, for $b>a, l(b)-l(a)=(b-a) p c^{p-1}$, where $c \in(a, b)$. Applying this to the first two terms in equation (5.5), we have

$$
\begin{aligned}
L(v) & \geq\left[\left(X_{0}+v^{2}\right)^{2}-F^{2}\right] p F^{2 p-2}-b v^{3}(3 b v / 2+\gamma) \\
& =v^{4}\left(p F^{2 p-2}-3 b^{2} / 2\right)-\gamma b v^{3}+2 X_{0} p F^{2 p-2} v^{2}+\left(X_{0}^{2}-F^{2}\right) p F^{2 p-2} \\
& \geq v^{2}\left[v^{2}\left(p F^{2 p-2}-3 b^{2} / 2\right)-\gamma b v+2 X_{0} p F^{2 p-2}\right]=v^{2} M(v),
\end{aligned}
$$

where we have used $X_{0}>F$ to obtain the last inequality. Hence, we need to show that for $b>0$ sufficiently small, $M(v) \geq 0$ for $v \geq 0$. First, write $M(v)=a_{2} v^{2}-a_{1} b v+a_{0}, a_{1}, a_{0}>0$, and let $b$ be sufficiently small that $a_{2}>0$. Let $\tilde{M}(v)=M(v) / a_{2}=v^{2}-2 c_{1} b v+c_{0}$, with $c_{1}, c_{0}>0$. Then $\tilde{M}(v)=\left(v-b c_{1}\right)^{2}+c_{0}-b^{2} c_{1}^{2}$, and it is plain that $b$ can be chosen to be small enough that $\tilde{M}(v)$, and so $L(v)$, are non-negative for $v \geq 0$.

We will also require $\mathcal{S}\left(-X_{0}\right)$ to extend to infinite negative $y$ values; it has this property provided that the curves $y=-b\left(-X_{0}-x\right)^{3 / 2}$ and $y=\left(F^{2 p}-x^{2 p}\right) / \gamma$ nowhere intersect for $y \leq 0$. This, too, is clearly true if $b$ is small enough.

It is possible that some of the conditions applied in the above proof could be relaxed, but a 'better' invariant set $\mathcal{S}$ is not required in the proof of the following theorem.

Theorem 4 For all $\boldsymbol{x}_{0}=\left(x_{0}, y_{0}\right) \in \mathcal{J}, \exists-X_{0} \leq-\xi$ which is such that $\boldsymbol{x}_{0} \in \mathcal{S}\left(-X_{0}\right)$. All solutions starting from such an $\boldsymbol{x}_{0}$ blow up in finite time.

Proof. Let $\boldsymbol{x}_{0}=(x(0), y(0))=\left(x_{0}, y_{0}\right) \in \mathcal{J}$. Then there always exists an $-X_{0} \leq-\xi$ such that $\boldsymbol{x}_{0}$ is in a subset $\mathcal{S}\left(-X_{0}\right)$ of $\mathcal{J}$ : choose any $-X_{0}>x_{0}$. Also, since $\dot{x} \leq 0$ in $\mathcal{J}, x \leq x_{0}$ and so $-X_{0}-x \geq-X_{0}-x_{0}>$ 0 . Additionally, since $\boldsymbol{x}_{0} \in \mathcal{S}\left(-X_{0}\right)$, the differential inequality $-b\left(-X_{0}-x\right)^{3 / 2} \geq y=\dot{x} \geq\left(F^{2 p}-x^{2 p}\right) / \gamma$ applies for all time $t \geq 0$, by the invariance of $\mathcal{S}\left(-X_{0}\right)$.

In fact, only the upper bound is important here, and with the substitution $u=-X_{0}-x>0$ and $u_{0}=-X_{0}-x_{0}>0$, this becomes $\dot{u} \geq b u^{3 / 2}$. Integrating gives

$$
\int_{u_{0}}^{u} \mathrm{~d} \psi \psi^{-3 / 2} \geq b \int_{0}^{t} \mathrm{~d} s,
$$

giving $u_{0}^{-1 / 2}-u^{-1 / 2} \geq b t / 2$, which, after re-arranging, gives

$$
u \geq\left[u_{0}^{-1 / 2}-b t / 2\right]^{-2} \quad \text { for } t \in\left[0, t_{\infty}\right)
$$

where $b t_{\infty}=2 u_{0}^{-1 / 2}$. The above inequality shows that $x(t)$ tends to $-\infty$ within a finite time $t \leq t_{\infty}$. By the invariance of $\mathcal{S}\left(-X_{0}\right), x(t)$ cannot tend to $-\infty$ without also $y(t) \rightarrow-\infty$, and so finite time blow up is proven.

Note that for $p=1$ there is a set $\mathcal{B}$ (cf. figure 3 ) such that all solutions starting from $\mathcal{B}$ enter $\mathcal{J}$ in a finite time: this was proved in [3]. Therefore for $p=1$ Theorem 4 shows that all trajectories starting from $\mathcal{B}$ blow up in finite time. 


\section{Open problems}

The construction of the basin of attraction of the quasi-periodic attractor given in sections 3 and 4 is far better than that of [1]. Still, in principle it is not optimal, and one could ask whether appreciable improvements could be obtained.

Also, for dissipation large enough, numerically all solutions which do dot blow up are found to be attracted by the quasi-periodic solution which has the same frequency vector as the forcing. Hence the orbit of such a solution seems to be the only attractor for $\gamma$ large enough. However, an analytical proof of this result is lacking.

Note that, in any case, one cannot expect that the set $\mathcal{B}^{c}$ and the basin of attraction of the quasiperiodic attractor are complementary to each other. In fact, the latter depends on the initial phase, whereas the former describes the set of initial data in the phase plane $(x, \dot{x})$ which blow up independently of their initial phase. Hence the phase plane, as well as the two sets $\mathcal{B}^{c}$ and the basin of attraction, contains a further set of initial data, whose behaviour is determined by the initial phase: according to what that is, the corresponding trajectories can either collapse onto the quasi-periodic attractor or diverge (in finite time, by Theorem 4). Whether these are the two only possibilities is still an open problem: at the moment, we can only say that the numerics strongly suggest that this is the case.

\section{References}

[1] M.V. Bartuccelli, J.H.B. Deane, G. Gentile, Globally and locally attractive solutions for quasiperiodically forced systems, J. Math. Anal. Appl., to appear.

[2] M.V. Bartuccelli, J.H.B. Deane, G. Gentile, S. Gourley, Global attraction to the origin in a parametrically driven nonlinear oscillator, Appl. Math. Comput. 153 (2004), no. 1, 1-11.

[3] M.V. Bartuccelli, J.H.B. Deane, G. Gentile, L. Marsh, Invariant sets for the varactor equation, R. Soc. Lond. Proc. Ser. A Math. Phys. Eng. Sci. 462 (2006), no. 2066, 439-457.

[4] G. Gentile, M.V. Bartuccelli, J.H.B. Deane, Summation of divergent series and Borel summability for strongly dissipative equations with periodic or quasi-periodic forcing terms, J. Math. Phys. 46 (2005), no. 6, 062704, 21 pp.

[5] G. Gentile, M.V. Bartuccelli, J.H.B. Deane, Quasi-periodic attractors, Borel summability and the Bryuno condition for strongly dissipative systems, J. Math. Phys. 47 (2006), no. 7, 072702, 10 pp.

[6] N.N. Krasovskiü, Stability of motion. Applications of Lyapunov's second method to differential systems and equations with delay, Stanford University Press, Stanford, Calif., 1963. 